\title{
Integrability and WKB solution of twist-three evolution equations.
}

\author{
A.V. Belitsky円 \\ Institut für Theoretische Physik, Universität Regensburg \\ D-93040 Regensburg, Germany
}

\begin{abstract}
We identify an integrable one-dimensional inhomogeneous three-site open spin chain which arises in the problem of diagonalization of twist-three quark-gluon evolution equations in QCD in the chiral-odd sector. Making use of the existence of a non-trivial 'hidden' integral of motion the problem of diagonalization of the evolution kernels is reduced to the study of a second order finite-difference equation which is solved in WKB approximation for large conformal spins of the three-particle system. The energies (alias anomalous dimensions) of eigenstates with different scale dependence are found in limiting cases and compared with numerical calculations.
\end{abstract}

Keywords: twist-three operators, evolution, three-particle problem, integrability, open spin chain, spectrum of eigenvalues

PACS numbers: 11.10.Gh, 11.15.Kc, 11.30.Na, 12.38.Cy

\footnotetext{
${ }^{1}$ Alexander von Humboldt Fellow.
} 


\section{Brief phenomenological profile and outline.}

Deep inelastic scattering of leptons off nucleons, $\ell N \rightarrow \ell^{\prime} X$, served for a long time as the only and the most reliable piece of knowledge about the partonic structure of hadrons in high energy collisions via measurements of non- and polarized chiral-even structure functions [1]. However the feasibility of measurements of other inclusive characteristics of hadrons was strongly limited. Nowadays in order to study in detail the high-energy dynamics of constituents, especially in polarized targets, one is forced to use complementary sources of experimental information. In order to circumvent the above disadvantages of conventional deep inelastic scattering process one has to resort to nucleon-nucleon collisions, like the Drell-Yan lepton pair production $h h^{\prime} \rightarrow$ $\left(\gamma^{*}, W, Z\right) \rightarrow \ell^{+} \ell^{-} X$, which are not affected by the above restraints and allow to explore a wealth of hadronic properties, e.g. to access a new type of parton densities - chiral odd distributions $h_{1}$ at twist- $2, h_{L}$ and $e$ at twist-3, and, related by QCD equation of motion to the latter two, generalized three-parton correlators [2]. The twist-3 distributions being a direct manifestation of the quark-gluon interaction in non-perturbative domain could serve as a testing ground for the phenomenological models of confinement and, perhaps, future lattice simulations.

Similar to the more familiar chiral-even transverse spin structure function $g_{2}, h_{L}$ although being of higher twist turns out to be unique by its feature to appear as a leading unsuppressed effect in longitudinal-transverse asymmetry in $p_{\|} p_{\perp} \rightarrow \ell^{+} \ell^{-} X$ reaction [2, 31. However, an inevitable complication of all experiments is that the data is taken at different values of a hard momentum transfer $Q$ and, therefore, to deduce the shape of distributions or to check sum rules one needs to know the way to describe the variation of quantities in question when going up(down)wards with $Q$ from the point of view of the underlying microscopic theory of strong interaction Quantum Chromodynamics. Within the latter framework this change is described by evolution equations which in turn arise as a result of intrinsic ultraviolet divergencies of the product of local elementary fields, - which define an operator content of distributions, - separated by light cone distances, and thus require renormalization which introduces a scale dependence. The specifics of twist-3 sector as compared to the well-studied twist- 2 case is that the above mentioned structure functions being defined as two-particle hadron-hadron matrix elements receive contributions from the three-parton quark-gluon correlators [4, 国]. Resolving this complication in favour of a study of the renormalization group evolution of the latter one ends up with a very complicated problem of working out the mixing of three-particle local operators which in general cannot be handled analytically.

One can ague that it is not really possible to see this logarithmic violation of scaling experimentally due to the contamination of a more strong $1 / Q$ background behaviour of prefactors which accompany the asymmetries. Nevertheless this knowledge is important for contrasting the 
theoretical models with the measurable quantities. Since the model estimations are usually done at a rather low momentum scale the evolution effects are very prominent and change in a sizable way the shape of an input distribution during short evolution times. So that perturbative evolution can be thought of as a prime mover of gross features of non-perturbative functions at hadronic scales, however much larger then $\Lambda_{\mathrm{QCD}}$. Recall in this respect the radiatively generated parton densities [6].

The distribution functions $h_{L}$ and $e$ as measured in most of processes are given, up to 'kinematical' Wandzura-Wilczek-type contribution in $h_{L}$ which obeys the ordinary twist-2 evolution, by particular integrals (see Eqs. (2,3) below) of three-particle quark-gluon correlation functions. The first drastic simplification of their evolution occurs for large number of colours. In multicolour limit the weight in the integrand of convolution integrals coincides with the eigenfunction of the leading order evolution kernel and, therefore, the above mentioned chiral-odd structure functions evolve multiplicatively — the so-called 'decoupling' phenomenon [7, 8, 9]. However, in the spin-independent Drell-Yan process the three-particle correlators associated with the unpolarized distribution $e$ enter folded with different coefficient functions [2] and, therefore, will not evolve autonomously even in multicolour limit. In the realistic QCD case, when the $1 / N_{c}^{2}$ correction are accounted for, the structure of evolution becomes more complicated: all three-particle operators are of relevance. But the violation of the simple pattern of multicolour evolution can be well understood once it is the case for large- $N_{c}$ limit. Since these corrections produce $\propto(10-20) \%$ effects in the evolution of the model distributions? which although being small and apparently might be sufficient for practical purposes at present should still be under control. This paper is devoted to an analysis of the large- $N_{c}$ spectrum of the three-particle evolution equations.

Our consequent presentation is organized as follows: In the next section we describe an approach to study the scale violation of the so-called quasi-partonic operators which are related to the quantities in question by QCD equations of motion. We show that the tree-level conformal symmetry of classical QCD Lagrangian allows for a partial diagonalization of the evolution kernels. In section 3 we elaborate on this point further and construct an appropriate three-particle conformal basis. A distinguishing property of the problem at hand is its equivalence to an exactly solvable one-dimensional open spin chain which is established in section 4. Making use of the existence of a 'hidden' integral of motion allows to reduce the eigenvalue problem for the energy of the system (alias anomalous dimensions) to the solution of a second order finite-difference equation - referred to as the master equation — which is derived in section 5. Consequent discussion concerns the study of the analytical structure of the spectrum of the conserved charge for large

\footnotetext{
${ }^{2}$ We discard from consideration some pathological shapes of gluon distribution in three-parton correlators which induce non-smooth evolution [11].
} 
values of the total conformal spin. Its asymptotic solutions are given in section 6 . These results allow to describe fairly well the spectrum of energy eigenvalues found by numerical diagonalization of the mixing matrix of the anomalous dimensions. Finally, we go to conclusions.

\section{Approach to scaling violation phenomena.}

In QCD the twist-3 distributions, $h_{L}$ and $e$, are expressed via the light-cone Fourier transformation of bilocal quark string operators [2]

$$
\left(\begin{array}{c}
e(x) \\
h_{L}(x)
\end{array}\right)=\frac{x}{2} \int \frac{d \kappa}{2 \pi} e^{i \kappa x}\left\langle h\left|\bar{\psi}(0) \Phi[0, \kappa n]\left(\begin{array}{c}
1 \\
i \sigma_{+-}
\end{array}\right) \psi(\kappa n)\right| h\right\rangle,
$$

with a phase factor $\Phi[0, \kappa n]$ which ensures the non-abelian gauge invariance. Although these definitions resemble the ones of ordinary twist-2 parton densities a more closer inspection immediately reveals an essential difference from the latter: twist-3 functions involve the "bad" light-cone components of the fields in the language of the Kogut-Soper infinite momentum frame formalism [10]. By virtue of the QCD Heisenberg equation of motion for the quark field and constraints coming from Lorentz invariance, the functions (1) can be rewritten in an explicitly interaction dependent way as 11

$$
\begin{aligned}
e(x) & =\int \frac{d \beta}{x-\beta} Z(x, \beta), \\
h_{L}(x) & =2 x^{2} \int_{x}^{1} \frac{d \beta}{\beta^{2}} h_{1}(\beta)+x^{2} \int_{x}^{1} \frac{d \beta}{\beta^{2}} \int \frac{d \beta^{\prime}}{\beta^{\prime}-\beta}\left[\frac{\partial}{\partial \beta}-\frac{\partial}{\partial \beta^{\prime}}\right] \widetilde{Z}\left(\beta, \beta^{\prime}\right) .
\end{aligned}
$$

in terms of correlation functions

$$
Z\left(x_{1}, x_{2}=-x_{1}-x_{3}, x_{3}\right)=\int \frac{d \kappa_{1}}{2 \pi} \frac{d \kappa_{3}}{2 \pi} e^{i \kappa_{1} x_{1}+i \kappa_{3} x_{3}}\left\langle h\left|\frac{1}{2}\left\{\mathcal{Z}\left(\kappa_{1}, 0, \kappa_{3}\right) \pm \mathcal{Z}\left(-\kappa_{3}, 0,-\kappa_{1}\right)\right\}\right| h\right\rangle
$$

of the non-local quark-gluon operators

$$
\left(\begin{array}{c}
\mathcal{Z} \\
\widetilde{\mathcal{Z}}
\end{array}\right)\left(\kappa_{1}, \kappa_{2}, \kappa_{3}\right)=\frac{1}{2} \bar{\psi}\left(\kappa_{3} n\right) \sigma_{\mu+}^{\perp}\left(\begin{array}{c}
1 \\
\gamma_{5}
\end{array}\right) t^{a} g G_{+\mu}^{a \perp}\left(\kappa_{2} n\right) \psi\left(\kappa_{1} n\right),
$$

with gauge link factors omitted for brevity. However the operators in Eq. (5), while contain one parton more, share a bulk of properties with the usual twist-2 operators: only "good" light-cone components and collinear momenta appear. "Bad" fields and transverse momentum dependence have been eliminated in favour of interaction which originates them. Thus in this description the theory is endowed again with a parton-like interpretation albeit of the QCD improved quark

\footnotetext{
${ }^{3}$ The first contribution on the r.h.s. of Eq. (3) is afore mentioned 'kinematical' twist-2 WW-term [2].
} 


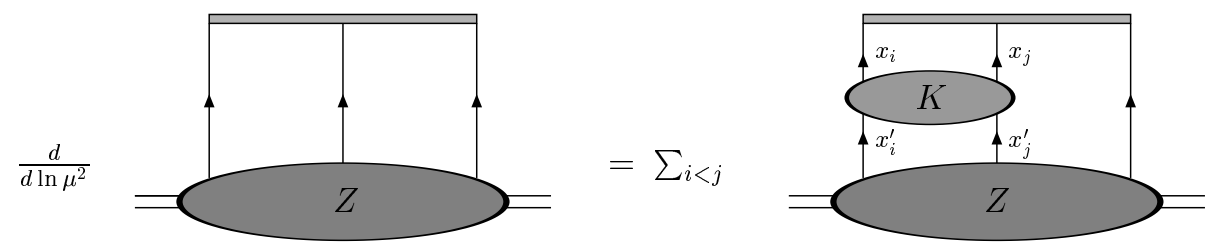

Figure 1: The diagrammatical representation of the generic form of the leading order evolution equation for the three-parton correlator $Z\left(x_{1}, x_{2}, x_{3}\right)$ in the light-cone gauge. The blob $K$ stands for the pair-wise kernel of interaction of $t$-channel particles.

model. And these are the properties which distinguish a special class of the so-called quasi-partonic operators 12 which are the most appropriate for the study of renormalization group dependence as the corresponding evolution equation turns out to be homogeneous. This latter property has arisen due to the fact that the pairs of parton fields in Eq. (5) correspond to the familiar twist-2 operators although they can have fermionic quantum numbers besides bosonic.

\subsection{Evolution equation.}

The generic form of the evolution equation for twist-3 correlation functions in the momentum fraction formalism looks like (see Fig. 1)

$$
\mu^{2} \frac{d}{d \mu^{2}} Z\left(x_{1}, x_{2}, x_{3}\right)=\int \prod_{i=1}^{3} d x_{i}^{\prime} \delta\left(\sum_{i=1}^{3} x_{i}^{\prime}\right) \boldsymbol{K}\left(\left\{x_{i}\right\} \mid\left\{x_{i}^{\prime}\right\}\right) Z\left(x_{1}^{\prime}, x_{2}^{\prime}, x_{3}^{\prime}\right),
$$

with the constraint $x_{1}+x_{2}+x_{3}=0$ of the forward inclusive kinematics for the momentum fractions of partons imposed on the both sides of this equation. Due to topology of one-loop Feynman diagrams the evolution kernel $\boldsymbol{K}$ has a simple pair-wise structure at leading order:

$$
\boldsymbol{K}\left(\left\{x_{i}\right\} \mid\left\{x_{i}^{\prime}\right\}\right)=\sum_{i<j} K\left(x_{i}, x_{j} \mid x_{i}^{\prime}, x_{j}^{\prime}\right) \delta\left(x_{i}+x_{j}-x_{i}^{\prime}-x_{j}^{\prime}\right)
$$

with $K\left(x_{i}, x_{j} \mid x_{i}^{\prime}, x_{j}^{\prime}\right)$ being a twist-2 interaction kernel of two nearby particles.

The solution to the leading order evolution equation (6) can be written in the form

$$
Z\left(x_{1}, x_{2}, x_{3} \mid Q^{2}\right)=\sum_{\{\alpha\}} \Psi_{\{\alpha\}}\left(x_{1}, x_{2}, x_{3}\right)\left(\frac{\alpha_{s}\left(Q_{0}^{2}\right)}{\alpha_{s}\left(Q^{2}\right)}\right)^{2 \mathcal{E}_{\{\alpha\}} / \beta_{0}}\left\langle\left\langle\mathcal{Z}_{\{\alpha\}}\left(Q_{0}^{2}\right)\right\rangle\right\rangle
$$

of expansion w.r.t. polynomials $\Psi_{\{\alpha\}}\left(x_{1}, x_{2}, x_{3}\right)$ which are the eigenfunctions of the leading order kernel (7) with eigenvalues $\mathcal{E}_{\{\alpha\}}$ and where $\left\langle\left\langle\mathcal{Z}_{\{\alpha\}}\right\rangle\right\rangle$ denote reduced hadronic matrix elements of local operators defined as (Mellin-like) moments of the three-parton correlation function. The summation goes over a set of quantum numbers $\{\alpha\}$ which enumerate the solutions, e.g. spin of the system etc. And $\beta_{0}=\frac{4}{3} T_{F} N_{f}-\frac{11}{3} C_{A}$ is the first coefficients of the QCD Gell-Mann-Low function. 
Since the polynomials depending on the momentum fractions can be immediately translated by Fourier transform to the ones depending on derivatives acting on the elementary field operators, they will correspond to the eigenvectors of the anomalous dimension matrix in a basis of local operators. Thus the problem of solving the evolution equation is equivalent to the diagonalization of the matrix of anomalous dimensions

$$
\boldsymbol{K} \Psi_{\{\alpha\}}=-\frac{\alpha_{s}}{2 \pi} \mathcal{E}_{\{\alpha\}} \Psi_{\{\alpha\}},
$$

where we have explicitly extracted the dependence on the coupling constant $\alpha_{s}$.

For the problem at hand the total evolution kernel takes the same form for the parity-even $(e)$ and -odd $\left(h_{L}\right)$ sectors

$$
\begin{aligned}
\boldsymbol{K}\left(\left\{x_{i}\right\} \mid\left\{x_{i}^{\prime}\right\}\right)=-\frac{\alpha_{s}}{2 \pi} & \left\{{ }^{q \bar{q}} K_{(8)}^{T}\left(x_{1}, x_{3} \mid x_{1}^{\prime}, x_{3}^{\prime}\right) \delta\left(x_{1}+x_{3}-x_{1}^{\prime}-x_{3}^{\prime}\right)\right. \\
& +{ }^{q g} K_{(3)}^{V}\left(x_{1}, x_{2} \mid x_{1}^{\prime}, x_{2}^{\prime}\right) \delta\left(x_{1}+x_{2}-x_{1}^{\prime}-x_{2}^{\prime}\right) \\
& \left.+{ }^{q g} K_{(3)}^{V}\left(x_{3}, x_{2} \mid x_{3}^{\prime}, x_{2}^{\prime}\right) \delta\left(x_{3}+x_{2}-x_{3}^{\prime}-x_{2}^{\prime}\right)-\frac{\beta_{0}}{4} \delta\left(x_{1}-x_{1}^{\prime}\right) \delta\left(x_{2}-x_{2}^{\prime}\right)\right\},
\end{aligned}
$$

with known non-forward evolution kernels, ${ }^{\phi_{1} \phi_{2}} K_{(c)}^{D}$, for colour triplet $(c=3)$ vector $(D=V)$ and colour octet $(c=8)$ transversity $(D=T)$ sectors. The selection of particular solutions comes from different parity properties of three-variable functions $Z$ and $\widetilde{Z}$ w.r.t. permutation of quark fields: the former is symmetrical $Z\left(x_{1}, x_{2}\right)=Z\left(x_{2}, x_{1}\right)$ while the latter is antisymmetrical $\widetilde{Z}\left(x_{1}, x_{2}\right)=-\widetilde{Z}\left(x_{2}, x_{1}\right)$ under this interchange. We have added in Eq. (10) the trivial charge renormalization counterterm which is traced back to the presence of the coupling constant in the definition of the composite operator (5).

\subsection{Eigenvalue problem.}

Due to absence of the QCD $\beta$-function contribution in leading order pair-wise evolution kernels we can use the conformal invariance property of QCD at tree level since the renormalization counterterms of composite operators at leading order are just the ones in classical theory while from the side of QCD Lagrangian the only counterterms which are required in the physical sector to cure the divergencies are the field renormalization constants. But the anomalous dimensions associated to them can be embedded into redefined representations of the conformal group replacing the canonical by the scale field dimensions - and thus preserve conformal covariance.

Making use of this property of classical QCD one can diagonalize the pair-wise non-forward kernels [13- 18] and present them in a factorized form [19]

$$
{ }^{\phi_{1} \phi_{2}} K\left(x_{1}, x_{2} \mid x_{1}^{\prime}, x_{2}^{\prime}\right)=\frac{1}{2} \sum_{j=0}^{\infty} \frac{w\left(x_{1}, \nu_{1} \mid x_{2}, \nu_{2}\right)}{\omega_{j}\left(\nu_{1}, \nu_{2}\right)} P_{j}^{\left(\nu_{2}, \nu_{1}\right)}\left(\frac{x_{1}-x_{2}}{x_{1}+x_{2}}\right){ }^{\phi_{1} \phi_{2}} \gamma_{j} P_{j}^{\left(\nu_{2}, \nu_{1}\right)}\left(\frac{x_{1}^{\prime}-x_{2}^{\prime}}{x_{1}^{\prime}+x_{2}^{\prime}}\right),
$$


where $w\left(x_{1}, \nu_{1} \mid x_{2}, \nu_{2}\right)=x_{1}^{\nu_{1}} x_{2}^{\nu_{2}}$ and $\omega_{j}\left(\nu_{1}, \nu_{2}\right)=\frac{\Gamma\left(j+\nu_{1}+1\right) \Gamma\left(j+\nu_{2}+1\right)}{\left(2 j+\nu_{1}+\nu_{2}+1\right) j ! \Gamma\left(j+\nu_{1}+\nu_{2}+1\right)}$, with $\nu_{\ell}=d_{\ell}+s_{\ell}-1$, and $d_{\ell}$ and $s_{\ell}$ standing for the canonical dimension and spin of the constituent $\phi_{\ell}=\{q, g\}$. The $P_{j}^{\left(\nu_{2}, \nu_{1}\right)}$ are the usual Jacobi polynomials 20] which form an infinite dimensional representation of the collinear conformal group [21], — whose $s u(1,1)$ algebra

$$
\left[\mathcal{J}^{3}, \mathcal{J}^{ \pm}\right]_{-}= \pm \mathcal{J}^{ \pm}, \quad\left[\mathcal{J}^{+}, \mathcal{J}^{-}\right]_{-}=-2 \mathcal{J}^{3}
$$

is defined by generators of the momentum $\mathcal{J}^{+}=i \mathcal{P}_{+}$, special conformal transformation $\mathcal{J}^{-}=\frac{i}{2} \mathcal{K}_{-}$ and particular combination of dilatation and angular momentum $\mathcal{J}^{3}=\frac{i}{2}\left(\mathcal{D}+\mathcal{M}_{-+}\right)$, - in the space spanned by bilinear (in elementary field operators) local operators with total derivatives [13. 16

$$
\mathcal{O}_{j k}=\phi_{2}\left(i \partial_{1}+i \partial_{2}\right)^{k} P_{j}^{\left(\nu_{2}, \nu_{1}\right)}\left(\frac{\partial_{1}-\partial_{2}}{\partial_{1}+\partial_{2}}\right) \phi_{1} .
$$

These operators possess conformal spin $J_{12}=j+\frac{1}{2}\left(\nu_{1}+\nu_{2}+2\right)$ which is the eigenvalue $\left[\mathcal{J}^{2}, \mathcal{O}_{j k}\right]_{-}=$ $J_{12}\left(J_{12}-1\right) \mathcal{O}_{j k}$ of the Casimir operator $\mathcal{J}^{2}=\mathcal{J}^{3}\left(\mathcal{J}^{3}-1\right)-\mathcal{J}^{+} \mathcal{J}^{-}$.

In the Eq. (11), all dynamics of the system is concentrated in the anomalous dimensions of the conformal operators, ${ }^{\phi_{1} \phi_{2}} \gamma_{j}$. Thus the former depend only on the eigenvalues of the two-particle Casimir operators in subchannels. For the eigenvalue problem (9) it can be translated to the fact that the pair-wise kernels commute with generators of conformal transformation, thus, again being a function of Casimir operator only.

This dependence can be easily traced from the corresponding eigenvalues. For our consequent considerations we need the bosonic colour-octet chiral-odd, ${ }^{q \bar{q}} \gamma_{(8) j}^{T}$ and fermionic colour-triplet chiral-evenf ${ }^{q g} \gamma_{(3)_{j}}^{V}$ anomalous dimensions which read

$$
\begin{aligned}
{ }^{q \bar{q}} \gamma_{(8) j}^{T}= & \left(C_{F}-\frac{C_{A}}{2}\right)(4 \psi(j+2)-4 \psi(1)-3) \\
{ }^{q g} \gamma_{(3) j}^{V}= & \frac{C_{A}}{2}(2 \psi(j+1)+2 \psi(j+4)-4 \psi(1)) \\
& \quad+\left(C_{F}-\frac{C_{A}}{2}\right) \frac{4 \sigma(j)}{(j+1)(j+2)(j+3)}+\frac{1}{4}\left(\beta_{0}-3 C_{A}\right),
\end{aligned}
$$

where $\sigma(j)=(-1)^{j}$.

Therefore, the evolution equation can be reformulated into the eigenvalue problem for the three-particle system in a basis of local operators

$$
\mathcal{H}_{\mathrm{QCD}} \Psi=\mathcal{E}_{\mathrm{QCD}} \Psi
$$

where $\boldsymbol{K} \equiv-\frac{\alpha_{s}}{2 \pi} \mathcal{H}$ with Hamiltonian

$$
\mathcal{H}_{\mathrm{QCD}}={ }^{q \bar{q}} \mathcal{H}_{(8)}^{T}\left(\hat{J}_{13}\right)+{ }^{q 9} \mathcal{H}_{(3)}^{V}\left(\hat{J}_{12}\right)+{ }^{q 9} \mathcal{H}_{(3)}^{V}\left(\hat{J}_{32}\right)-\frac{\beta_{0}}{4},
$$

\footnotetext{
${ }^{4}$ We do not bother about parity since it is irrelevant for fermionic operators.
} 
and we have defined ${ }^{q \bar{q}} \gamma_{J-2}=2^{q \bar{q}} \mathcal{H}(J)$ and ${ }^{q g} \gamma_{J-5 / 2}=2^{q \mathscr{H}} \mathcal{H}(J)$. The operators $\hat{J}$ 's are formally determined as solutions of the operator equation for Casimir operator in some representation? $\hat{\boldsymbol{J}}^{2}=\hat{J}(\hat{J}-1)$.

One of the complications to find the solution of the eigenvalue problem (16) is a rather nontrivial dependence on the colour structure of the quark-gluon system. In order to get rid of it we take large $N_{c}$ limit in Eq. (17) which results into omission of the $1 / N_{c}^{2}$ terms in Eq. (14). This leads to the Hamiltonian

$$
\mathcal{H}=\frac{N_{c}}{2}\left\{h_{12}+h_{23}-\frac{3}{2}\right\}, \quad \text { with } \quad h_{a b}=\psi\left(\hat{J}_{a b}-\frac{3}{2}\right)+\psi\left(\hat{J}_{a b}+\frac{3}{2}\right)-2 \psi(1),
$$

whose diagonalization will be our cherished goal.

\section{Conformal basis: General formalism.}

As we have seen above the conformal covariance provides a partial diagonalization of the threeparton evolution kernels. Therefore, an appropriate basis constructed with regards to the tree-level conformal symmetry can simplify the problem further. This question is considered below in the present section.

\subsection{Auxiliary $\theta$-space.}

It turns out to be convenient to introduce an auxiliary vector space where the conformal invariance properties are reformulated in a manifest and simple way. This description appears once we consider the elements

$$
\theta^{k} \equiv \frac{\partial_{+}^{k} \phi}{\Gamma(k+\nu+1)}
$$

as building blocks of the formalism. In this representation $\left[\mathcal{J}^{ \pm, 3}, \chi(\theta)\right]_{-}=\hat{J}^{ \pm, 3} \chi(\theta)$ the generators take the following form

$$
\hat{J}^{+}=(\nu+1) \theta+\theta^{2} \frac{\partial}{\partial \theta}, \quad \hat{J}^{-}=\frac{\partial}{\partial \theta}, \quad \hat{J}^{3}=\frac{1}{2}(\nu+1)+\theta \frac{\partial}{\partial \theta},
$$

and the quadratic Casimir operator is given by

$$
\hat{\boldsymbol{J}}^{2}=\hat{J}^{3}\left(\hat{J}^{3}-1\right)-\hat{J}^{+} \hat{J}^{-} .
$$

${ }^{5}$ Note that the operators $\hat{J}$ introduced here differs from the ones defined in Ref. 24 by a shift: $\hat{J}^{\text {here }}=\hat{J}^{24}+1$.

${ }^{6}$ Once conformal invariance is established we can abstract from the particular representation used for the derivation. 
For a multi-variable function $\chi\left(\theta_{1}, \theta_{2}, \ldots, \theta_{n}\right)$ the operators are defined by the sum of single particle ones as $\hat{J}^{ \pm, 3}=\sum_{\ell=1}^{n} \hat{J}_{\ell}^{ \pm, 3}$ and they obey the usual commutation relations: $\left[\hat{J}^{3}, \hat{J}^{ \pm}\right]_{-}= \pm \hat{J}^{ \pm}$, $\left[\hat{J}^{+}, \hat{J}^{-}\right]_{-}=-2 \hat{J}^{3}$. Obviously, the single particle state $\theta_{\ell}$ is an eigenstate of the Casimir operator

$$
\hat{\boldsymbol{J}}_{\ell}^{2} \theta_{\ell}=\hat{\boldsymbol{\jmath}}_{\ell}^{2} \theta_{\ell}, \quad \text { with } \quad \hat{\boldsymbol{\jmath}}_{\ell}^{2} \equiv \frac{1}{4}\left(\nu_{\ell}^{2}-1\right)
$$

Since the Eq. (16) defines the physical anomalous dimensions, the latter have to be real that requires the Hamiltonian (18) to be selfadjoint] w.r.t. an appropriate scalar product. We define the latter as

$$
\left\langle\chi\left(\theta_{1}, \theta_{2}, \ldots, \theta_{n}\right) \mid \chi\left(\theta_{1}, \theta_{2}, \ldots, \theta_{n}\right)\right\rangle=\int_{\Omega} d \mathcal{M} \chi\left(\bar{\theta}_{1}, \bar{\theta}_{2}, \ldots, \bar{\theta}_{n}\right) \chi\left(\theta_{1}, \theta_{2}, \ldots, \theta_{n}\right)
$$

where

$$
d \mathcal{M} \equiv \prod_{\ell=1}^{n} \frac{d \theta_{\ell} d \bar{\theta}_{\ell}}{2 \pi i}\left(1-\theta_{\ell} \bar{\theta}_{\ell}\right)^{\nu_{\ell}-1} \quad \text { and } \quad \Omega=\bigcup_{\ell=1}^{n}\left\{\left|\theta_{\ell}\right| \leq 1\right\}
$$

and $\bar{\theta}=\theta^{*}$. Then it can be seen that the Casimir operator and, as a consequence, the Hamiltonian are selfadjoint operators w.r.t. such defined inner product

$$
\left(\hat{\boldsymbol{J}}^{2}\right)^{\dagger}=\hat{\boldsymbol{J}}^{2}, \quad \mathcal{H}^{\dagger}=\mathcal{H}
$$

and therefore $\operatorname{Im} \mathcal{E}=0$.

\subsection{Fundamental basis.}

Using the above definitions one can construct a second order differential operator (here and throughout $\left.\theta_{a b} \equiv \theta_{a}-\theta_{b}\right)$

$$
\hat{\boldsymbol{J}}_{a b}^{2}=-\theta_{a b}^{1-\left(\nu_{a}+\nu_{b}\right) / 2} \partial_{a} \partial_{b} \theta_{a b}^{1+\left(\nu_{a}+\nu_{b}\right) / 2}+\frac{1}{2}\left(\nu_{b}-\nu_{a}\right) \theta_{a b}\left(\partial_{a}+\partial_{b}\right)
$$

which corresponds to the two-particle Casimir operator in $(a, b)$-subchannel. Its eigenstates $\left(\hat{J}_{12}^{+}\right)^{k} \theta_{12}^{j}$ coincide with bilinear conformal operators (13) and possess the same eigenvalues. The three-particle Casimir is an obvious generalization

$$
\hat{\boldsymbol{J}}^{2}=\hat{\boldsymbol{J}}_{12}^{2}+\hat{\boldsymbol{J}}_{23}^{2}+\hat{\boldsymbol{J}}_{13}^{2}-\sum_{\ell=1}^{3} \hat{\boldsymbol{\jmath}}_{\ell}^{2}
$$

As it has been advocated in Refs. [22]-24] it proves convenient to introduce the following basis of three-particle operators which simultaneously diagonalize the total three-particle Casimir

\footnotetext{
${ }^{7}$ To be precise Hermitean matrices do possess real eigenvalues, however, the inverse statement in not true. Recall
} in this respect triangular matrices with real diagonal elements. 
operator $\hat{\boldsymbol{J}}^{2}$ and one in a subchannel $\hat{\boldsymbol{J}}_{a b}^{2}$, say $a=1$ and $b=2$,

$$
\begin{aligned}
\hat{\boldsymbol{J}}^{2} \mathcal{P}_{J ; j}\left(\theta_{1}, \theta_{2} \mid \theta_{3}\right) & =\left(J+\frac{1}{2}\left(\nu_{1}+\nu_{2}+\nu_{3}+3\right)\right)\left(J+\frac{1}{2}\left(\nu_{1}+\nu_{2}+\nu_{3}+1\right)\right) \mathcal{P}_{J ; j}\left(\theta_{1}, \theta_{2} \mid \theta_{3}\right), \\
\hat{\boldsymbol{J}}_{12}^{2} \mathcal{P}_{J ; j}\left(\theta_{1}, \theta_{2} \mid \theta_{3}\right) & =\left(j+\frac{1}{2}\left(\nu_{1}+\nu_{2}\right)\right)\left(j+\frac{1}{2}\left(\nu_{1}+\nu_{2}+2\right)\right) \mathcal{P}_{J ; j}\left(\theta_{1}, \theta_{2} \mid \theta_{3}\right) .
\end{aligned}
$$

The states with unit norm w.r.t. the scalar product (23) are defined immediately from the above equations and read

$$
\mathcal{P}_{J ; j}\left(\theta_{1}, \theta_{2} \mid \theta_{3}\right)=\mathcal{N}^{-1}\left(J, j \mid \nu_{1}, \nu_{2}, \nu_{3}\right) \sum_{k=0}^{J-j} \frac{(-1)^{k}\left(\begin{array}{c}
J-j \\
k
\end{array}\right)}{\left(2 j+\nu_{1}+\nu_{2}+2\right)_{k}} \theta_{3}^{J-j-k}\left(\hat{J}_{12}^{+}\right)^{k} \theta_{12}^{j}
$$

with normalization factor

$$
\mathcal{N}^{2}\left(J, j \mid \nu_{1}, \nu_{2}, \nu_{3}\right)=\frac{j !(J-j) !\left(j+\nu_{1}+\nu_{2}+1\right)_{j}}{\left(\nu_{1}\right)_{j+1}\left(\nu_{2}\right)_{j+1}\left(\nu_{3}\right)_{J-j+1}} \frac{\left(J+j+\nu_{1}+\nu_{2}+\nu_{3}+2\right)_{J-j}}{\left(2 j+\nu_{1}+\nu_{2}+2\right)_{J-j}},
$$

where we have introduced the Pochhammer symbol $(\alpha)_{\ell}=\Gamma(\alpha+\ell) / \Gamma(\alpha)$. By construction the above basis is orthonormal $\left\langle\mathcal{P}_{J^{\prime} ; j^{\prime}}\left(\theta_{1}, \theta_{2} \mid \theta_{3}\right) \mid \mathcal{P}_{J ; j}\left(\theta_{1}, \theta_{2} \mid \theta_{3}\right)\right\rangle=\delta_{J^{\prime} J} \delta_{j^{\prime} j}$.

\subsection{Matrix elements.}

The form of the basis given by Eq. (28) is especially suitable for evaluation of matrix elements of $S U(1,1)$ generators. The 'proper' generators of the basis $\mathcal{P}\left(\theta_{1}, \theta_{2} \mid \theta_{3}\right)$ are diagonal (27,26) and the only operators which possess off-diagonal elements are $\hat{\boldsymbol{J}}_{(1,2) 3}^{2}$. The important property of the basis (28) which distinguishes it among other possibilities is that the matrix elements of 'improper' operators are three-diagonal only

$$
\left\langle\mathcal{P}_{J^{\prime} ; j^{\prime}}\left(\theta_{1}, \theta_{2} \mid \theta_{3}\right)\left|\hat{\boldsymbol{J}}_{23}^{2}\right| \mathcal{P}_{J ; j}\left(\theta_{1}, \theta_{2} \mid \theta_{3}\right)\right\rangle=\delta_{J^{\prime} J}\left(\delta_{j^{\prime} j}\left[\hat{\boldsymbol{J}}_{23}^{2}\right]_{j, j}+\delta_{j^{\prime}, j+1}\left[\hat{\boldsymbol{J}}_{23}^{2}\right]_{j+1, j}+\delta_{j^{\prime}, j-1}\left[\hat{\boldsymbol{J}}_{23}^{2}\right]_{j-1, j}\right)
$$

where the diagonal part is

$$
\left[\hat{\boldsymbol{J}}_{23}^{2}\right]_{j, j}=\frac{1}{2}\left(\left[\hat{\boldsymbol{J}}^{2}\right]_{j j}-\left[\hat{\boldsymbol{J}}_{12}^{2}\right]_{j j}+\sum_{\ell=1}^{3} \hat{\boldsymbol{\jmath}}_{\ell}^{2}+\left(\hat{\boldsymbol{J}}_{2}^{2}-\hat{\boldsymbol{\jmath}}_{1}^{2}\right)\left(\left[\hat{\boldsymbol{J}}^{2}\right]_{j j}-\hat{\boldsymbol{\jmath}}_{3}^{2}\right)\left[\hat{\boldsymbol{J}}_{12}^{2}\right]_{j j}^{-1}\right),
$$

and the non-diagonal elements are

$$
\left[\hat{\boldsymbol{J}}_{23}^{2}\right]_{j+1, j}=\frac{\mathcal{N}\left(J, j \mid \nu_{1}, \nu_{2}, \nu_{3}\right)}{\mathcal{N}\left(J, j+1 \mid \nu_{1}, \nu_{2}, \nu_{3}\right)}(j+1)\left(J-j+\nu_{3}\right) .
$$

From hermiticity it follows that $\left[\hat{\boldsymbol{J}}_{23}^{2}\right]_{j+1, j}=\left[\hat{\boldsymbol{J}}_{23}^{2}\right]_{j, j+1}$. 


\subsection{Other representations of the basis.}

Since the operator (28) is the highest weight of the conformal group in the three-particle basis, i.e. $\hat{J}^{-} \mathcal{P}=0$, and is the homogeneous polynomial (through Eq. (26)) of degree $J$ by the Euler theorem, this suggests that the net dependence of Eq. (28) is on a single variable only (up to a prefactor). This is really the case and immediately one can perform the resummation of the series in Eq. (28) to obtain a compact expression for the $\theta$-space basis (cf. 23])

$$
\mathcal{P}_{J ; j}\left(\theta_{1}, \theta_{2} \mid \theta_{3}\right)=\mathcal{N}^{-1}\left(J, j \mid \nu_{1}, \nu_{2}, \nu_{3}\right) \theta_{12}^{J} \theta_{2}^{j-J} F_{1}\left(\begin{array}{c}
j-J, j+\nu_{1}+1 \\
2 j+\nu_{1}+\nu_{2}+2
\end{array} \mid \theta\right), \quad \text { with } \quad \theta \equiv \frac{\theta_{12}}{\theta_{32}} .
$$

In general the solution to the condition $\hat{J}^{-} \mathcal{P}=0$ is given by a translation invariant polynomial,

e.g. $\mathcal{P} \propto \sum_{j_{1}+j_{2}=J} c_{j_{1}, j_{2}} \theta_{12}^{j_{1}} \theta_{32}^{j_{2}}$ with undetermined expansion coefficients. This is the standard choice which leads to Appel polynomials when transformed back to the space of local operators [16. If, as we have done above, we will impose an additional constraint (27) we get a recursion relation for c's which when solved gives (33).

The relation to the space of local operators can be readily figure out by using the definition (19) so that after some algebra we end up with (cf. [23])

$$
\begin{gathered}
\phi_{2}\left(i \partial_{1}+i \partial_{2}\right)^{j} P_{j}^{\left(\nu_{2}, \nu_{1}\right)}\left(\frac{i \partial_{1}-i \partial_{2}}{i \partial_{1}+i \partial_{2}}\right) \phi_{1}\left(i \partial_{1}+i \partial_{2}+i \partial_{3}\right)^{J-j} P_{J-j}^{\left(2 j+\nu_{1}+\nu_{2}+1, \nu_{3}\right)}\left(\frac{i \partial_{3}-i \partial_{1}-i \partial_{2}}{i \partial_{1}+i \partial_{2}+i \partial_{3}}\right) \phi_{3} \\
=i^{J} \frac{\Gamma\left(j+\nu_{1}+1\right) \Gamma\left(j+\nu_{2}+1\right)}{\Gamma(j+1)} \\
\times \frac{\Gamma\left(J-j+\nu_{3}+1\right)}{\Gamma(J-j+1)} \frac{\Gamma\left(J+j+\nu_{1}+\nu_{2}+2\right)}{\Gamma\left(2 j+\nu_{1}+\nu_{2}+2\right)} \mathcal{N}\left(J, j \mid \nu_{1}, \nu_{2}, \nu_{3}\right) \mathcal{P}_{J ; j}\left(\theta_{1}, \theta_{2} \mid \theta_{3}\right) .
\end{gathered}
$$

\subsection{Racah decomposition.}

Since the choice of the second condition (27) on the basis is arbitrary, — one can analogously take a requirement for the element of the basis to be an eigenstate of operator $\hat{\boldsymbol{J}}_{(1,2) 3}^{2}$, — one poses the question as to how the transformation from one basis to another is accomplished. Obviously, we meet a standard quantum mechanical problem of addition of three 'angular momenta'. According to general theory they are related via the decomposition

$$
P_{13} \mathcal{P}_{J ; j}\left(\theta_{1}, \theta_{2} \mid \theta_{3}\right)=\mathcal{P}_{J ; j}\left(\theta_{3}, \theta_{2} \mid \theta_{1}\right)=\sum_{k=0}^{J} W_{j k}(J) \mathcal{P}_{J ; k}\left(\theta_{1}, \theta_{2} \mid \theta_{3}\right),
$$

where the coefficients $W_{j k}(J)$ are viewed as Racah symbols for $S U(1,1)$. The operator $P_{13}$ permutes the fields ' 1 ' and ' 3 ' in teh operators $\mathcal{P}_{J ; j}$. The explicit form of $W_{j k}(J)$ can be easily deduced from the above formula since ${ }^{\S} W_{j k}(J)=\left\langle\mathcal{P}_{J ; k}\left(\theta_{1}, \theta_{2} \mid \theta_{3}\right) \mid \mathcal{P}_{J ; j}\left(\theta_{3}, \theta_{2} \mid \theta_{1}\right)\right\rangle$ and making use

${ }^{8}$ The $W_{j k}(J)$ are the matrix elements of the operator $P_{13}$. 
of the representation (34) with the derivatives loosely interpreted as momentum fractions. Going to the special system with $i \partial_{1}=z, i \partial_{2}=1-z$ and $i \partial_{3}=0$, where the calculation methods of Ref. [18 can be applied in a straightforward way, we end up with the result

$$
\begin{aligned}
W_{j k}(J) & =(-1)^{J-k} \frac{\Gamma(J-j+1)}{\Gamma(J-k+1) \Gamma(k-j+1)} \frac{\Gamma\left(J+k+\nu_{1}+\nu_{2}+\nu_{3}+2\right)}{\Gamma\left(J+j+\nu_{1}+\nu_{2}+\nu_{3}+2\right)} \\
& \times \frac{\Gamma\left(k+\nu_{1}+1\right) \Gamma\left(k+\nu_{2}+1\right) \Gamma\left(k+\nu_{1}+\nu_{2}+1\right)}{\Gamma\left(j+\nu_{2}+1\right) \Gamma\left(2 k+\nu_{1}+\nu_{2}+1\right) \Gamma\left(2 k+\nu_{1}+\nu_{2}+2\right)} \\
& \times \frac{\Gamma\left(J+k+\nu_{1}+\nu_{2}+2\right) \Gamma\left(2 j+\nu_{2}+\nu_{3}+2\right)}{\Gamma\left(J-j+\nu_{1}+1\right) \Gamma\left(j+k+\nu_{2}+\nu_{3}+2\right)} \frac{\mathcal{N}\left(J, \nu_{1}, \nu_{2}, \nu_{3}\right)}{\mathcal{N}\left(J, j \mid \nu_{3}, \nu_{2}, \nu_{1}\right)} \\
& \times{ }_{4} F_{3}\left(\begin{array}{c}
-J+k, J+k+\nu_{1}+\nu_{2}+\nu_{3}+2, k+\nu_{2}+1, k+1 \\
j+k+\nu_{2}+\nu_{3}+2,2 k+\nu_{1}+\nu_{2}+2, k-j+1
\end{array}\right) .
\end{aligned}
$$

These coefficients are real $W_{j k}=W_{j k}^{*}$ and orthogonal

$$
\sum_{\ell=0}^{J} W_{j \ell} W_{k \ell}=\delta_{j k}
$$

They satisfy the three-term recursion relation

$$
\begin{aligned}
\left(j+\frac{\nu_{2}+\nu_{3}}{2}\right) & \left(j+\frac{\nu_{2}+\nu_{3}}{2}+1\right) W_{j k}(J) \\
= & {\left[\hat{\boldsymbol{J}}_{23}^{2}\right]_{k, k} W_{j k}(J)+\left[\hat{\boldsymbol{J}}_{23}^{2}\right]_{k, k-1} W_{j, k-1}(J)+\left[\hat{\boldsymbol{J}}_{23}^{2}\right]_{k, k+1} W_{j, k+1}(J) }
\end{aligned}
$$

with matrix elements of the Casimir operator $\hat{\boldsymbol{J}}_{23}^{2}$ evaluated above. Using it one can easily derive useful approximate formula in the limits $\tau \equiv \frac{k}{J}=$ fixed, $j=$ fixed, $J \rightarrow \infty$, to be used later. At leading order in $J$ Eq. (38) can be reduced to the second order differential equation the solution to which is given by

$$
w_{j}(\tau)=\sqrt{2}\left[\frac{\tau^{1+2 \nu_{2}}\left(1-\tau^{2}\right)^{\nu_{3}}}{\omega_{j}\left(\nu_{3}, \nu_{2}\right)}\right]^{1 / 2} P_{j}^{\left(\nu_{3}, \nu_{2}\right)}\left(2 \tau^{2}-1\right),
$$

whereg $W_{j k}=\frac{1}{\sqrt{J}}(-1)^{J-k} w_{j}(\tau)$.

\section{Three-site open spin chain and integrability.}

As we have established in the preceding sections the original problem of calculation the spectrum of the evolution equation (6) is reduced in multicolour limit to the diagonalization of the Hamiltonian (18) which defines the one-dimensional open spin chain with three sites. Moreover, the operators $\hat{\boldsymbol{J}}^{2}$ and $\hat{J}^{3}$ are shown to be the integrals of motion of the system, i.e. $\left[\left(\hat{\boldsymbol{J}}^{2}, \hat{J}^{3}\right), \mathcal{H}\right]_{-}=0$. In

\footnotetext{
${ }^{9}$ Here and below the powers of factor of $J$ in front of functions in continuous limit are introduced in order to have appropriate scaling properties for $J \rightarrow \infty$ when $\sum_{j=0}^{J} \rightarrow J \int_{0}^{1} d \tau$.
} 
addition to that there exists another 'hidden' conserved charge [22] which thus leads to an exact integrability of the three-particle system: the number of integrals of motion equals the number of degrees of freedom. Let us show that the system described by the Hamiltonian (18) is equivalent to an integrable one-dimensional open spin chain with impurities. For these purposes we will use the Quantum Inverse Scattering Method (QISM) formalism. The latter has been used earlier to prove the integrability of the multi-reggeon interaction in QCD relevant for the high-energy behaviour of physical cross sections and identify the corresponding model with the XXX Heisenberg magnet of non-compact $\operatorname{spin} s=0$ [25, 26].

The central object in the algebraic operator formalism of the QISM [27, 28] is the $R$-matrix which depends on a complex spectral parameter $\lambda$ and acts on the tensor product of vector spaces $V_{a} \otimes V_{b}$ having the dimensions of local spaces on each site. The indices $a$ and $b$ refer to the quantum and auxiliary spaces, respectively. The $R$-matrix obeys the star-triangle Yang-Baxter equation 27, 28

$$
R_{a, b}(\lambda-\mu) R_{a, c}(\lambda) R_{b, c}(\mu)=R_{b, c}(\mu) R_{a, c}(\lambda) R_{a, b}(\lambda-\mu) .
$$

In our consequent considerations we only discuss the three-site open spin chain. The monodromy matrix for it is defined as usual by

$$
T_{b}(\lambda)=R_{a_{1}, b}\left(\lambda-\delta_{1}\right) R_{a_{2}, b}\left(\lambda-\delta_{2}\right) R_{a_{3}, b}\left(\lambda-\delta_{3}\right),
$$

where the impurities, $\delta_{i}$, are introduced in order to enrich the family of representations. The transfer matrix for open spin chain [29]

$$
\hat{t}_{b}(\lambda)=\operatorname{tr}_{b} K^{+}(\lambda) T_{b}(\lambda) K^{-}(\lambda) T_{b}^{-1}(-\lambda)
$$

with trace taken over the auxiliary space, - has been shown by Sklyanin to form a family of mutually commuting operators 29]

$$
\left[\hat{t}_{b_{1}}\left(\lambda_{1}\right), \hat{t}_{b_{2}}\left(\lambda_{2}\right)\right]_{-}=0
$$

This is a direct consequence of the Yang-Baxter equation. The boundary matrices in Eq. (42) satisfy the reflection Sklyanin equations [29] and their simplest solution which leads to the conformal invariant transfer matrix is given by the unit matrix 四 $K^{ \pm}=\mathbb{1}$.

For the two-dimensional auxiliary space (corresponding to spin $\frac{1}{2}$ ) the $R$-matrix defines the Lax operator

$$
L_{a}(\lambda) \equiv R_{a, \frac{1}{2}}\left(\lambda-\frac{1}{2}\right)=\lambda \mathbb{1}+\sigma^{i} \hat{J}_{a}^{i}=\left(\begin{array}{cc}
\lambda+\hat{J}_{a}^{3} & \hat{J}_{a}^{-} \\
-\hat{J}_{a}^{+} & \lambda-\hat{J}_{a}^{3}
\end{array}\right),
$$

\footnotetext{
${ }^{10}$ Note that similar choice was made in the context of open spin chains arisen in the problem of integrability of quark-gluon reggeon equations [30].
} 
with $\hat{J}^{ \pm}=\mp\left(\hat{J}^{1} \pm i \hat{J}^{2}\right)$. The auxiliary transfer matrix which generates the set of local integrals of motion can be redefined according to [29] as

$$
\hat{t}_{\frac{1}{2}}\left(\lambda-\frac{1}{2}\right)=\prod_{\ell=1}^{3} \Delta^{-1}\left\{R_{a_{\ell}, \frac{1}{2}}\left(\lambda-\delta_{\ell}-1\right)\right\} t_{\frac{1}{2}}(\lambda)
$$

where $\Delta\left\{R_{a_{\ell}, \frac{1}{2}}(\lambda)\right\}=\lambda(\lambda+1)-\hat{\boldsymbol{\jmath}}_{\ell}^{2}$ stands for the quantum determinant [31, 32] and

$$
t_{\frac{1}{2}}(\lambda)=\operatorname{tr}_{\frac{1}{2}} L_{a_{1}}\left(\lambda-\delta_{1}\right) L_{a_{2}}\left(\lambda-\delta_{2}\right) L_{a_{3}}\left(\lambda-\delta_{3}\right) \sigma_{2} L_{a_{3}}^{\mathrm{t}}\left(-\lambda-\delta_{3}\right) L_{a_{2}}^{\mathrm{t}}\left(-\lambda-\delta_{2}\right) L_{a_{1}}^{\mathrm{t}}\left(-\lambda-\delta_{1}\right) \sigma_{2}
$$

which is an even function of the spectral parameter [29] $t_{\frac{1}{2}}(-\lambda)=t_{\frac{1}{2}}(\lambda)$. The expansion of this expression w.r.t. the rapidity, $\lambda$, defines the set of commuting integrals of motion.

For our purposes it is enough to restrict to the following values of the shifts: $\delta_{1}=\delta_{3} \equiv \delta$, $\delta_{2}=0$. An explicit calculation leads to the result

$$
t_{\frac{1}{2}}(\lambda)=\Omega(\lambda)-4 \lambda^{2}\left(\lambda^{2}-\hat{\boldsymbol{\jmath}}_{2}^{2}\right) \hat{\boldsymbol{J}}^{2}-2 \lambda^{2} \mathcal{Q}
$$

where $\Omega(\lambda)$ is a c-number function

$$
\Omega(\lambda)=-2\left(\lambda^{2}+\hat{\boldsymbol{\jmath}}_{1}^{2}-\delta^{2}\right)\left(\lambda^{2}+\hat{\boldsymbol{\jmath}}_{2}^{2}\right)\left(\lambda^{2}+\hat{\boldsymbol{\jmath}}_{3}^{2}-\delta^{2}\right)+4 \lambda^{2}\left(\lambda^{2}-\delta^{2}\right) \sum_{\ell=1}^{3} \hat{\boldsymbol{\jmath}}_{\ell}^{2}+4 \lambda^{2}\left(\hat{\boldsymbol{\jmath}}_{1}^{2} \hat{\boldsymbol{\jmath}}_{3}^{2}-\delta^{2} \hat{\boldsymbol{\jmath}}_{2}^{2}\right),
$$

$\hat{\boldsymbol{J}}^{2}$ is the total Casimir operator 25) and $\mathcal{Q}$ is a non-trivial integral of motion

$$
\mathcal{Q}=\left[\hat{\boldsymbol{J}}_{12}^{2}, \hat{\boldsymbol{J}}_{23}^{2}\right]_{+}-2 \delta^{2}\left\{\hat{\boldsymbol{J}}_{12}^{2}+\hat{\boldsymbol{J}}_{23}^{2}\right\} \text {. }
$$

The fundamental Hamiltonians which commutes with the above set of charges can be constructed following Sklyanin [29]. The fundamental monodromy matrix is defined by product (41) of $R$-matrices with equivalent representations of conformal spin in quantum and auxiliary spaces and the shifts of spectral parameters defined as above. Then the Hamiltonian of the chain is defined as

$$
\mathcal{H}=\left.\frac{1}{2} \frac{d}{d \lambda}\right|_{\lambda=0} \ln \hat{t}_{b}(\lambda)
$$

Due to the diagonal form of the boundary matrices the latter is given, up to irrelevant c-number term, by the sum of two-site Hamiltonians 29.

$$
\mathcal{H}=h_{a_{1} a_{2}}+h_{a_{2} a_{3}},
$$

which are expressed by the equation

$$
h_{a b}=\left.\frac{d}{d \lambda}\right|_{\lambda=0} \ln R_{a, b}(\lambda-\delta)=R_{a, b}^{-1}(-\delta) R_{a, b}^{\prime}(-\delta) .
$$


Making use of the explicit form of the Yang-Baxter bundle [33, 34]

$$
R_{a, b}(\lambda)=P_{a b} \frac{\Gamma\left(\hat{J}_{a b}+\lambda\right)}{\Gamma\left(\hat{J}_{a b}-\lambda\right)},
$$

one easily obtains

$$
h_{a b}=\psi\left(\hat{J}_{a b}-\delta\right)+\psi\left(\hat{J}_{a b}+\delta\right)-2 \psi(1)
$$

where we have legitimately added a c-number term to mimic the QCD pair-wise Hamiltonian (18).

The commutativity of $\mathcal{Q}(48)$ and $\mathcal{H}(50)$ can be checked a posteriori by an explicit calculation of the commutators $\left[\mathcal{Q}, h_{(1,3) 2}\right]_{-}$, e.g. in the conformal basis $(28)$. Here the problem is reduced to the evaluation of the only matrix element which reads

$$
\begin{aligned}
{\left[\mathcal{Q}, h_{12}\right]_{j, j+1} } & =\left\{\left[\hat{\boldsymbol{J}}_{12}^{2}\right]_{j+1, j+1}+\left[\hat{\boldsymbol{J}}_{12}^{2}\right]_{j, j}-2 \delta^{2}\right\}\left\{\left[h_{12}\right]_{j+1}-\left[h_{12}\right]_{j}\right\}\left[\hat{\boldsymbol{J}}_{23}^{2}\right]_{j, j+1} \\
& =2\left\{\left[\hat{\boldsymbol{J}}_{12}^{2}\right]_{j+1, j+1}-\left[\hat{\boldsymbol{J}}_{12}^{2}\right]_{j, j}\right\}\left[\hat{\boldsymbol{J}}_{23}^{2}\right]_{j, j+1},
\end{aligned}
$$

where in the last line we have used the explicit form of the matrix elements of the Hamiltonian (53). This result together with similar calculation for $\left[\mathcal{Q}, h_{23}\right]_{-}$in $P_{13}$-transformed basis gives

$$
\left[\mathcal{Q}, h_{12}\right]_{-}=2\left[\hat{\boldsymbol{J}}_{23}^{2}, \hat{\boldsymbol{J}}_{12}^{2}\right]_{-}, \quad\left[\mathcal{Q}, h_{23}\right]_{-}=2\left[\hat{\boldsymbol{J}}_{12}^{2}, \hat{\boldsymbol{J}}_{23}^{2}\right]_{-}
$$

and thus $[\mathcal{Q}, \mathcal{H}]_{-}=0$.

Finally, if we will put $\delta=\frac{3}{2}$ as suggested by QCD (18) we immediately obtain the conserved charge 'empirically' found in Ref. 22].

\section{Master equation.}

Thus as has been said above the existence of the additional charge $\mathcal{Q}$ leads to complete integrability of the quark-gluon system $\left(\nu_{1}=\nu_{3}=1, \nu_{2}=2\right)$. This implies that the Hamiltonian $\mathcal{H}$ is a function of the integrals of motion only, $\hat{\boldsymbol{J}}^{2}$ and $\mathcal{Q}$, and allows to reduce the complicated eigenvalue problem for the Hamiltonian to the more simple one for the charge $\mathcal{Q}_{T}$

$$
\mathcal{Q}_{T} \Psi=q_{T} \Psi \quad \text { with } \quad \mathcal{Q}_{T}=\left[\hat{\boldsymbol{J}}_{12}^{2}, \hat{\boldsymbol{J}}_{23}^{2}\right]_{+}-\frac{9}{2}\left\{\hat{\boldsymbol{J}}_{12}^{2}+\hat{\boldsymbol{J}}_{23}^{2}\right\}
$$

making use of the conformal invariance of the system. The latter condition means that it is convenient to look for the solution to (55) in the form of expansion w.r.t. the three-point basis (28) elaborated in the previous sections, i.e.

$$
\Psi=\sum_{j=0}^{J} \Psi_{j} \mathcal{P}_{J ; j} .
$$


The matrix elements of the charge possess, similar to the non-diagonal Casimir operators $\hat{\boldsymbol{J}}_{(1,2) 3}^{2}$, only three non-zero diagonals

$$
\left\langle\mathcal{P}_{J^{\prime} ; j^{\prime}}\left(\theta_{1}, \theta_{2} \mid \theta_{3}\right)\left|\mathcal{Q}_{T}\right| \mathcal{P}_{J ; j}\left(\theta_{1}, \theta_{2} \mid \theta_{3}\right)\right\rangle=\delta_{J^{\prime} J}\left(\delta_{j^{\prime} j}\left[\mathcal{Q}_{T}\right]_{j, j}+\delta_{j^{\prime}, j+1}\left[\mathcal{Q}_{T}\right]_{j+1, j}+\delta_{j^{\prime}, j-1}\left[\mathcal{Q}_{T}\right]_{j-1, j}\right)
$$

where

$$
\begin{aligned}
& {\left[\mathcal{Q}_{T}\right]_{j, j}=2\left[\hat{\boldsymbol{J}}_{12}^{2}\right]_{j j}\left[\hat{\boldsymbol{J}}_{23}^{2}\right]_{j j}-\frac{9}{2}\left\{\left[\hat{\boldsymbol{J}}_{12}^{2}\right]_{j j}+\left[\hat{\boldsymbol{J}}_{23}^{2}\right]_{j j}\right\},} \\
& {\left[\mathcal{Q}_{T}\right]_{j+1, j}=\left\{\left[\hat{\boldsymbol{J}}_{12}^{2}\right]_{j+1, j+1}+\left[\hat{\boldsymbol{J}}_{12}^{2}\right]_{j j}-\frac{9}{2}\right\}\left[\hat{\boldsymbol{J}}_{23}^{2}\right]_{j+1, j},}
\end{aligned}
$$

and $\left[\mathcal{Q}_{T}\right]_{j+1, j}=\left[\mathcal{Q}_{T}\right]_{j, j+1}$. Therefore, the above equation (55) leads to a three-term recursion relation which can written in a concise matrix form by introducing the two-dimensional vector $\boldsymbol{\Psi}_{j}=\left(\begin{array}{c}\Psi_{j} \\ \Psi_{j-1}\end{array}\right)$ as

$$
\boldsymbol{\Psi}_{j+1}=\boldsymbol{M}_{j} \boldsymbol{\Psi}_{j} \quad \text { with } \quad \boldsymbol{M}_{j}=\left(\begin{array}{cc}
-\frac{\left[\mathcal{Q}_{T}\right]_{j, j}-q_{T}}{\left[\mathcal{Q}_{T}\right]_{j, j+1}} & -\frac{\left[\mathcal{Q}_{T}\right]_{j, j-1}}{\left[\mathcal{Q}_{T}\right]_{j, j+1}} \\
1 & 0
\end{array}\right)
$$

It must be supplied with the boundary conditions $\Psi_{-1}=\Psi_{J+1}=0$ which manifest the polynomial character of the eigenfunctions. The solution to the Eq. (60) can be presented thus in the form

$$
\boldsymbol{\Psi}_{j+1}=\boldsymbol{M}_{j} \boldsymbol{M}_{j-1} \ldots \boldsymbol{M}_{0} \boldsymbol{\Psi}_{0}
$$

with the 'vacuum' state $\boldsymbol{\Psi}_{0}=\left(\begin{array}{l}1 \\ 0\end{array}\right)$ where we have voluntary assumed $\Psi_{0}=1$ due to its arbitrary normalization. The boundary condition $\Psi_{J+1}=0$ presumes that the $q_{T}$ values are the real roots of (yet unknown) orthogonal polynomials. For the expansion coefficients themselves it implies that the eigenvalues of the matrix $\boldsymbol{M}_{j}$ must be complex because otherwise the solution will be a monotonic function of $j$ on the whole interval $j \in[0, J]$ and the only way to fulfill the boundary condition is to accept necessarily the trivial solution $\Psi_{j}=0$. Therefore, from the eigenvalue equation, $\operatorname{det}[\boldsymbol{M}-M \cdot \mathbb{1}]=0$, we get the condition for existence of the non-trivial solution to the recursion relation

$$
\left(\left[\mathcal{Q}_{T}\right]_{j, j}-q_{T}\right)^{2} \leq 4\left[\mathcal{Q}_{T}\right]_{j, j+1}\left[\mathcal{Q}_{T}\right]_{j, j-1}
$$

Let us redefine the expansion coefficient according to

$$
\Psi_{j} \equiv \varrho_{j} \Upsilon_{j}
$$

where

$$
\varrho_{j}=\left[\frac{(j+1)^{3}(j+3)^{3}}{(j+2)^{3}}(J-j+1)(J+j+5)\right]^{-\frac{1}{2}} .
$$




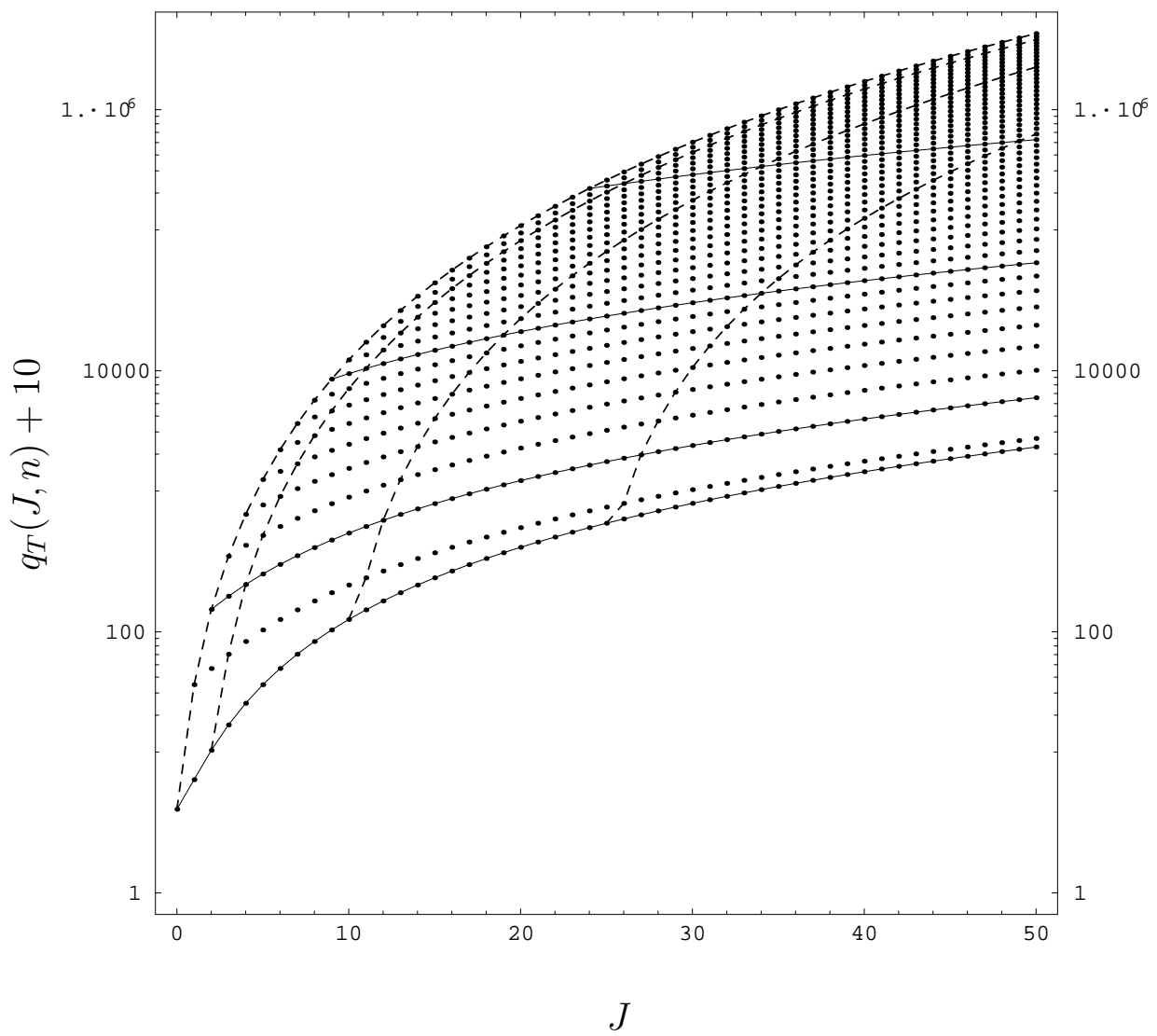

Figure 2: The spectrum of the conserved charge $q_{T}$ and a few trajectories from different sets (see the text).

The recursion relation then takes more transparent form which reads

$$
(2 j+3) \Upsilon_{j+1}+(2 j+5) \Upsilon_{j-1}+\varrho_{j}^{2}(2 j+3)(2 j+5)\left(\left[\mathcal{Q}_{T}\right]_{j, j}-q_{T}\right) \Upsilon_{j}=0 .
$$

This master equation will be the main object of our consequent analysis. Unfortunately, there is little hope to solve Eq. (64) analytically for arbitrary values of the total conformal spin, therefore, below we develop a WKB-type expansion for the conserved charge and the energy of the three-site chain at large $J$.

\section{Quantization of integral of motion.}

From the constraint (61) one can readily derive the critical values of the spectrum of the conserved charge for asymptotical values of the total conformal spin $J$. This leads to the inequality

$$
0 \leq q_{T} / J^{4} \leq \frac{1}{2}
$$

and to the fact that the maximum of the spectrum is achieved for $j_{\max }=\frac{1}{\sqrt{2}} J$. This means that in the vicinity of the upper boundary of the $q_{T}$-eigenvalues the coefficients $\Psi_{j}$ are peaked around this 
point. The validity of the above estimate can be checked by explicit numerical diagonalization of the matrix (57) which gives the quantized values of the charge $\mathcal{Q}_{T}$. The resulting spectrum is shown in Fig. 2. We can immediately notice that the values of $q_{T}$ form the families of oneparametric curves. Obviously, there are two possible sets of trajectories which apparently lead to an equivalent description of the spectrum. The first set which starts from the top of the spectrum (shown by dashed lines in Fig. 2) and behaves as

$$
q_{T}^{\mathrm{top}}=J^{4}\left(c_{0}^{\mathrm{top}}(n)+c_{1}^{\mathrm{top}}(n) J^{-1}+\mathcal{O}\left(J^{-2}\right)\right)
$$

These trajectories are labeled by an integer $n$. Comparing this expression with Eq. (65) we conclude that $c_{0}^{\text {top }}(n)=\frac{1}{2}$. Another alternative is to adhere to the parametrization of the curves starting from the bottom (shown by solid curves)

$$
q_{T}^{\text {bot }}=J^{2}\left(c_{0}^{\text {bot }}(m)+c_{1}^{\text {bot }}(m)[\ln J+a]^{-2}+\mathcal{O}\left(J^{-1}, \ln ^{-4} J\right)\right),
$$

labeled by $m$.

These results can be viewed as different analytical continuations of the charge, defined for integer $J$, to complex values of the conformal spin. Whatever choice we made, - that can be judged by employing external physical arguments, - the result is the same: the fine structure of the spectrum is generated by the pre-asymptotic corrections in the conformal spin $J$.

\subsection{Description from below.}

Let us address first the question of the description of the spectrum of the charge starting from below. In this case the trajectories behave as $q_{T} \propto J^{2}$ and one can immediately find two exact solutions to the master equation

$$
q_{T}^{\text {exact-1 }}(J)=-\frac{53}{8}+(J+1)^{2}, \quad q_{T}^{\text {exact-2 }}(J)=-\frac{53}{8}+(J+5)^{2},
$$

separated by a gap from the rest of the spectrum. At this boundary of $q_{T}$-values the classically allowed region, as deduced from Eq. (64), tends to spread over the whole interval of attained values of $j$ with exception of small vicinities of the end points $j_{\text {end }} \sim 1, J$.

To find the WKB solution let us introduce the function $\Upsilon_{j}=J(-1)^{j} \phi(\tau)$ with $j \equiv \tau J$. Then the recursion relation can be reduced in leading order in $J$ to the second order differential equation for $\phi(\tau)$ :

$$
\tau^{2}\left(1-\tau^{2}\right) \phi^{\prime \prime}(\tau)-\tau\left(1-\tau^{2}\right) \phi^{\prime}(\tau)+2\left(q_{T}^{\star}-1+4 \tau^{2}\right) \phi(\tau)=0,
$$

where $q_{T}^{\star}=J^{-2} q_{T}$. The solution to this equation is given by

$$
\phi(\tau)=C^{(+)} \tau^{1+2 i \eta_{T}}{ }_{2} \mathcal{F}_{1}\left(i \eta_{T} \mid \tau^{2}\right)+C^{(-)} \tau^{1-2 i \eta_{T}}{ }_{2} \mathcal{F}_{1}\left(-i \eta_{T} \mid \tau^{2}\right),
$$


with $C^{( \pm)}$being complex constants and ${ }_{2} \mathcal{F}_{1}\left( \pm i \eta_{T} \mid \tau^{2}\right)={ }_{2} F_{1}\left(\begin{array}{c}\frac{3}{2} \pm i \eta_{T},-\frac{3}{2} \pm i \eta_{T} \\ 1 \pm 2 i \eta_{T}\end{array} \mid \tau^{2}\right)$, where we have introduced the shorthand notation $\eta_{T} \equiv \frac{1}{2} \sqrt{2 q_{T}^{\star}-3}$.

To determine the unknown coefficients $C^{( \pm)}$one has to find the solution of the master equation in the vicinity of the deflection points $\tau \rightarrow 0,1$, i.e. $J \gg j \sim 1$ and $J \gg J-j \sim 1$, respectively.

For $J \gg J-j \sim 1$ the master equation simplifies into

$$
\Upsilon_{j+1}+\Upsilon_{j-1}+2 \Upsilon_{j}=0
$$

with boundary condition $\Upsilon_{J+1}=0$, and the solution to which is

$$
\Upsilon_{j}=(-1)^{j}(J-j+1)
$$

An important property of the solutions we have found above is that there exists a region, $J-j \sim$ $\sqrt{J}$, where both of them are valid so that by sewing them together we get the unknown coefficients $C^{( \pm)}$. Namely,

$$
\frac{C^{(+)}}{C^{(-)}}=-\frac{{ }_{2} \mathcal{F}_{1}\left(-i \eta_{T} \mid 1\right)}{{ }_{2} \mathcal{F}_{1}\left(i \eta_{T} \mid 1\right)} .
$$

The quantization condition for the charge $q_{T}$ can be obtained from the similar procedure used at the other end of values attained by $j$. However, instead of an explicit solution of the resulting reduced (but still quite complicated) recursion relation one can adopt the strategy based on the definite symmetry properties of the system under permutation of quark fields. Assuming the eigenfunction of the 'hidden' charge to be simultaneously the eigenfunctions of the permutation operator $P_{13}: P_{13} \Psi=e^{i \varphi} \Psi$ with $\varphi=0, \pi$ and $P_{13} \mathcal{P}_{J ; j}\left(\theta_{1}, \theta_{2} \mid \theta_{3}\right)=\mathcal{P}_{J ; j}\left(\theta_{3}, \theta_{2} \mid \theta_{1}\right)$; we get

$$
\Psi_{j}=e^{i \varphi} \sum_{k=0}^{J} W_{j k}(J) \Psi_{k}
$$

with the phase being the function of the conserved charge $q_{T}$ the dependence on which is determined by the relation

$$
\varphi=\arg (-1)^{J} \sum_{j=0}^{J}(-1)^{j} \frac{(j+2)^{3}}{(j+1)(j+3)} \Upsilon_{j} .
$$

Therefore, we can find the boundary solutions making use of known WKB ones. Thus for the region $J \gg j \sim 1$ we get immediately

$$
\Upsilon_{j}=(-1)^{J}\left[\frac{(j+1)(j+3)}{(j+2)}\right]^{3 / 2} e^{i \varphi} \int_{0}^{1} d \tau \frac{w_{j}(\tau) \phi(\tau)}{\left[\tau^{3}\left(1-\tau^{2}\right)\right]^{1 / 2}}
$$

After simple algebra it can be cast to the form

$$
\begin{aligned}
\Upsilon_{j} & =(-1)^{J+j} \frac{(j+1)(j+3)}{(j+2)} e^{i \varphi} \\
& \times\left\{C^{(+)}\left[(-1)^{j}{ }_{2} \mathcal{F}_{1}\left(i \eta_{T} \mid 1\right)+\frac{\Gamma\left(\frac{3}{2}+i \eta_{T}\right) \Gamma\left(\frac{5}{2}+j-i \eta_{T}\right)}{\Gamma\left(\frac{3}{2}-i \eta_{T}\right) \Gamma\left(\frac{5}{2}+j+i \eta_{T}\right)}{ }_{4} \mathcal{F}_{3}\left(i \eta_{T} \mid 1\right)\right]+(+\rightarrow-)\right\} .
\end{aligned}
$$


where ${ }_{4} \mathcal{F}_{3}\left(i \eta_{T} \mid 1\right)={ }_{4} F_{3}\left(\begin{array}{c}-\frac{3}{2}+i \eta_{T},-\frac{1}{2}+i \eta_{T}, \frac{3}{2}+i \eta_{T}, \frac{3}{2}+i \eta_{T} \\ -\frac{3}{2}-j+i \eta_{T}, \frac{5}{2}+j+i \eta_{T}, 1+2 i \eta_{T}\end{array} \mid 1\right)$ and possesses definite scaling properties when expressed by the series. Moreover, Eq. (75) obviously fulfills the boundary condition $\Upsilon_{-1}=0$.

From Eq. (68) it follows that for large $J$

$$
\Upsilon_{j}=(-1)^{j}\left\{C^{(+)} j^{1+2 i \eta_{T}} J^{-2 i \eta_{T}}+C^{(-)} j^{1-2 i \eta_{T}} J^{2 i \eta_{T}}\right\}\{1+\mathcal{O}(j / J)\}
$$

while from (75) we have

$$
\begin{aligned}
\Upsilon_{j}=(-1)^{J+j} e^{i \varphi}\{ & C^{(+)} j^{1-2 i \eta_{T}} \frac{\Gamma\left(\frac{3}{2}+i \eta_{T}\right)}{\Gamma\left(\frac{3}{2}-i \eta_{T}\right)}+C^{(-)} j^{1+2 i \eta_{T}} \frac{\Gamma\left(\frac{3}{2}-i \eta_{T}\right)}{\Gamma\left(\frac{3}{2}+i \eta_{T}\right)} \\
& \left.+(-1)^{j} j\left[C^{(+)}{ }_{2} \mathcal{F}_{1}\left(i \eta_{T} \mid 1\right)+C^{(-)}{ }_{2} \mathcal{F}_{1}\left(-i \eta_{T} \mid 1\right)\right]\right\}\{1+\mathcal{O}(1 / j)\} .
\end{aligned}
$$

Matching these equations in the region of their overlap $j \sim \sqrt{J}$ making use of Eq. (71) results into the following quantization condition for the charge

$$
2 \eta_{T} \ln J-\arg \frac{\Gamma\left(\frac{3}{2}+i \eta_{T}\right)}{\Gamma\left(\frac{3}{2}-i \eta_{T}\right)} \frac{\mathcal{F}_{1}\left(-i \eta_{T} \mid 1\right)}{{ }_{2} \mathcal{F}_{1}\left(i \eta_{T} \mid 1\right)}=\pi m
$$

with $m \in \mathbb{Z}_{+}$. The trajectories generated by this equation are the ones shown by solid lines in Fig. 2 .

\subsection{Description from above.}

For the description from above the classically allowed region is concentrated in the vicinity of the point $j_{\max }=\frac{1}{\sqrt{2}} J$. Therefore, we expand the master equation around it, $j=\frac{1}{\sqrt{2}}(J+\lambda \sqrt{J})$, and look for the solution to Eq. (64) in the form of series w.r.t. inverse powers of $J$

$$
\Phi(\lambda)=\sum_{\ell=0}^{\infty} \Phi_{(\ell)}(\lambda) J^{-\ell / 2}, \quad \text { and } \quad q_{T}(J, n)=J^{4} \sum_{\ell=0}^{\infty} q_{T}^{(\ell)}(n) J^{-\ell}
$$

where $\Upsilon_{j} \equiv \Phi(\lambda)$. This leads to the following sequence of differential equations

$$
\begin{aligned}
& \mathcal{D}_{(1)} \Phi_{(0)}(\lambda)=0 \\
& \mathcal{D}_{(1)} \Phi_{(1)}(\lambda)+\mathcal{D}_{(2)} \Phi_{(0)}(\lambda)=0 \\
& \mathcal{D}_{(1)} \Phi_{(2)}(\lambda)+\mathcal{D}_{(2)} \Phi_{(1)}(\lambda)+\mathcal{D}_{(3)} \Phi_{(0)}(\lambda)=0 \\
& \mathcal{D}_{(1)} \Phi_{(3)}(\lambda)+\mathcal{D}_{(2)} \Phi_{(2)}(\lambda)+\mathcal{D}_{(3)} \Phi_{(1)}(\lambda)+\mathcal{D}_{(4)} \Phi_{(0)}(\lambda)=0,
\end{aligned}
$$


supplied with boundary conditions $\Phi( \pm \infty)=0$ and explicit form of differential operators given by

$$
\begin{aligned}
& \mathcal{D}_{(1)}=\frac{d^{2}}{d \lambda^{2}}+4\left(6-q_{T}^{(1)}-2 \lambda^{2}\right), \\
& \mathcal{D}_{(2)}=-\frac{d}{d \lambda}+8 \lambda\left(6-4 \sqrt{2}-\lambda^{2}\right), \\
& \mathcal{D}_{(3)}=\frac{1}{6} \frac{d^{4}}{d \lambda^{4}}+4\left(3-\lambda^{2}\right) \frac{d^{2}}{d \lambda^{2}}+\lambda \frac{d}{d \lambda}-\left(43-96 \sqrt{2}+4 q_{T}^{(2)}-24 \lambda^{2}+48 \sqrt{2} \lambda^{2}+2 \lambda^{4}\right), \\
& \mathcal{D}_{(4)}=-\frac{1}{3} \frac{d^{3}}{d \lambda^{3}}+4 \lambda\left(6-4 \sqrt{2}-\lambda^{2}\right) \frac{d^{2}}{d \lambda^{2}}+\left(-12+2 \sqrt{2}+3 \lambda^{2}\right) \frac{d}{d \lambda}-2 \lambda\left(57-48 \sqrt{2}+8 \sqrt{2} \lambda^{2}\right), \\
& \ldots
\end{aligned}
$$

The solution to Eq. (80) is expressed by Hermite polynomials

$$
\Phi_{(0)}(\lambda)=H_{n}(\sqrt{2 \sqrt{2}} \lambda) e^{-\sqrt{2} \lambda^{2}} .
$$

The solutions to others (81) are given by

$$
\Phi_{(\ell)}(\lambda)=\int d \lambda^{\prime} G\left(\lambda, \lambda^{\prime}\right) \mathcal{J}_{(\ell)}\left(\lambda^{\prime}\right)+C_{(\ell)} \Phi_{(0)}(\lambda)
$$

with the Green function of the homogeneous equation (80), $\mathcal{D}_{(1)} G\left(\lambda, \lambda^{\prime}\right)=\delta\left(\lambda-\lambda^{\prime}\right)$, and the source $\mathcal{J}_{(\ell)}=-\sum_{k=0}^{\ell-1} \mathcal{D}_{(\ell+1-k)} \Phi_{(k)}$. Explicitly they are given by a linear combination of the Hermite polynomials, e.g. for the first non-trivial solution we have

$$
\Phi_{(1)}(\lambda)=\frac{e^{-\sqrt{2} \lambda^{2}}}{2 \sqrt{2 \sqrt{2}}} \sum_{k} c_{k} H_{k}(\sqrt{2 \sqrt{2}} \lambda),
$$

with non-zero coefficients $c_{n+3}=-\frac{1}{24}, c_{n+1}=-\frac{1}{4}(33-24 \sqrt{2}+3 n), c_{n-1}=n\left(17-12 \sqrt{2}+\frac{3}{2} n\right)$ and $c_{n-3}=\frac{1}{3} n(n-1)(n-2)$. Expressions for higher corrections are more involved.

The quantization for the charge $q_{T}^{(1)}$ immediately follows from boundary conditions. The quantization of higher WKB corrections, $q_{T}^{(\ell)}(\ell \geq 2)$, appears from the condition of absence of zero-mode in the source terms. In this way we derive a few next-to-leading corrections to the charge

$$
\begin{aligned}
q_{T}^{(0)}(n) & =\frac{1}{2} \\
q_{T}^{(1)}(n) & =6-\frac{1}{\sqrt{2}}-\sqrt{2} n, \\
q_{T}^{(2)}(n) & =\frac{1}{16}(371-72 \sqrt{2})+\frac{1}{8}(11-72 \sqrt{2}) n+\frac{11}{8} n^{2},
\end{aligned}
$$

(with $n=0,1, \ldots$ ) which allow to describe the upper part of the spectrum with a high accuracy. The integer $n$ has an obvious interpretation as the number of nodes of the solutions in the classically allowed region. 


\section{Energy.}

The main advantage of having the integrability of the system is that the eigenfunctions of integrals of motion simultaneously are the ones for the Hamiltonian. By taking the average of the Hamiltonian w.r.t. its eigenfunctions $\Psi$ we readily obtain

$$
\frac{2}{N_{c}} \mathcal{E}(J, n)=2\left(\sum_{j=0}^{J} \varrho_{j}^{2} \Upsilon_{j}^{2}\right)^{-1} \sum_{j=0}^{J} \epsilon(j) \varrho_{j}^{2} \Upsilon_{j}^{2}-\frac{3}{2}
$$

with $\epsilon(j)=\psi(j+1)+\psi(j+4)+2 \gamma_{E}$.

At the same time using the representation of the fundamental basis in terms of the hypergeometric function (33) and taking into account definite symmetry properties of the system under permutation of quarks one can derive the equation linear in the expansion coefficients

$$
\frac{2}{N_{c}} \mathcal{E}(J, n)=\left(\sum_{j=0}^{J}(-1)^{j} \frac{(j+2)^{3}}{(j+1)(j+3)} \Upsilon_{j}\right)^{-1} \sum_{j=0}^{J}(-1)^{j} \epsilon(j) \frac{(j+2)^{3}}{(j+1)(j+3)} \Upsilon_{j}+\frac{1}{3}
$$

Both of these formulae display the complementary aspects of the formalism. When supplied by the solution of the master equation they provide the exact solution of the three-body problem under study.

Thus in order to find the explicit form of the energy as a function of the conserved charges one has to substitute the WKB solutions found before into Eq. (86) or (87).

\subsection{Description from below.}

The lowest trajectories corresponding to the solutions (66) were known before [8, 35, 11]

$$
\begin{aligned}
\mathcal{E}^{\text {exact-1 }}(J) & =\frac{N_{c}}{2}\left\{2 \psi(J+3)+2 \gamma_{E}-\frac{1}{2}-\frac{1}{J+3}\right\} \\
\mathcal{E}^{\text {exact-2}}(J) & =\frac{N_{c}}{2}\left\{2 \psi(J+3)+2 \gamma_{E}-\frac{1}{2}+\frac{3}{J+3}\right\} .
\end{aligned}
$$

The first (second) energy corresponds to the anomalous dimension of the genuine twist-3 part of the structure function $e\left(h_{L}\right)$ in multicolour QCD since as has been found in the references alluded to above the eigenfunctions corresponding to Eq. (88) coincide with the coefficient function which enter into the relation between $e$ and $h_{L}$ and corresponding three-particle correlators (2, 3). Therefore, the description from below seems to have more physical grounds to be trusted as a true analytical continuation of the anomalous dimensions to complex $J$ since otherwise it looks dubious that the trajectories (88) pick out just one point for a given $J$ from another set of 'genuine' analytical functions discussed below in the next section 7.2 . 
The remainder of the spectrum can be found by inserting the expression for the eigenfunctions (68) with (71) into Eq. (87). We obtain the following result for the energy levels after the gap (cf. [22)

$$
\frac{2}{N_{c}} \mathcal{E}\left(J, \eta_{T}\right)=2 \ln J+4 \gamma_{E}+2 \operatorname{Re} \psi\left(\frac{3}{2}+i \eta_{T}\right)-\frac{3}{2} .
$$

As will be shown by numerical analysis this result is valid with high accuracy for the whole spectrum.

\subsection{Description from above.}

To evaluate the energy for the top of the spectrum it is more convenient to use the definition (86). The energy can be evaluated consistently in WKB approximation as expansion in inverse powers of the conformal spin $J$ as

$$
\mathcal{E}(J, n)=\frac{N_{c}}{2}\left\{\mathcal{E}^{(0)}(J)+\sum_{\ell=1}^{\infty} \mathcal{E}^{(\ell)}(n) J^{-\ell}\right\} .
$$

Substituting the solutions (82), (84) into (86) and equating the coefficients in front of $J^{-\ell}$ we get the leading and non-leading corrections to the energy

$$
\begin{aligned}
& \mathcal{E}^{(0)}(J)=4 \ln J+4 \gamma_{E}-2 \ln 2-\frac{3}{2}, \\
& \mathcal{E}^{(1)}(n)=12-\sqrt{2}-2 \sqrt{2} n, \\
& \ldots
\end{aligned}
$$

Thus at large $J$ the asymptotic behaviour of the energy of the three-site open spin chain is given by

$$
\frac{2}{N_{c}} \mathcal{E}\left(J, q_{T}\right)=\ln \left(q_{T} / 2\right)+4 \gamma_{E}-\frac{3}{2}+\mathcal{O}\left(J^{-2}\right) .
$$

And again as before in Eq. (89) the energy is a function of the conserved charges of the problem.

\subsection{Comparison with numerical analysis.}

Let us confront the analytical results derived so far with explicit numerical diagonalization of the QCD anomalous dimension matrix. This can be done either by using the expression of the Hamiltonian in the conformal basis elaborated before

$$
\frac{2}{N_{c}} \mathcal{H}_{j k}=\left(\epsilon(j)-\frac{3}{2}\right) \delta_{j k}+\sum_{\ell=0}^{J} W_{\ell j}(J) \epsilon(\ell) W_{\ell k}(J),
$$

or making use of known expression for the multicolour anomalous dimension matrix in the basis of ordinary local operators $\mathcal{Z}_{J ; j} \propto \bar{\psi}\left(i \partial_{+}\right)^{j} g G_{+\perp}\left(i \partial_{+}\right)^{J-j} \psi$ which is more suitable for numerical 


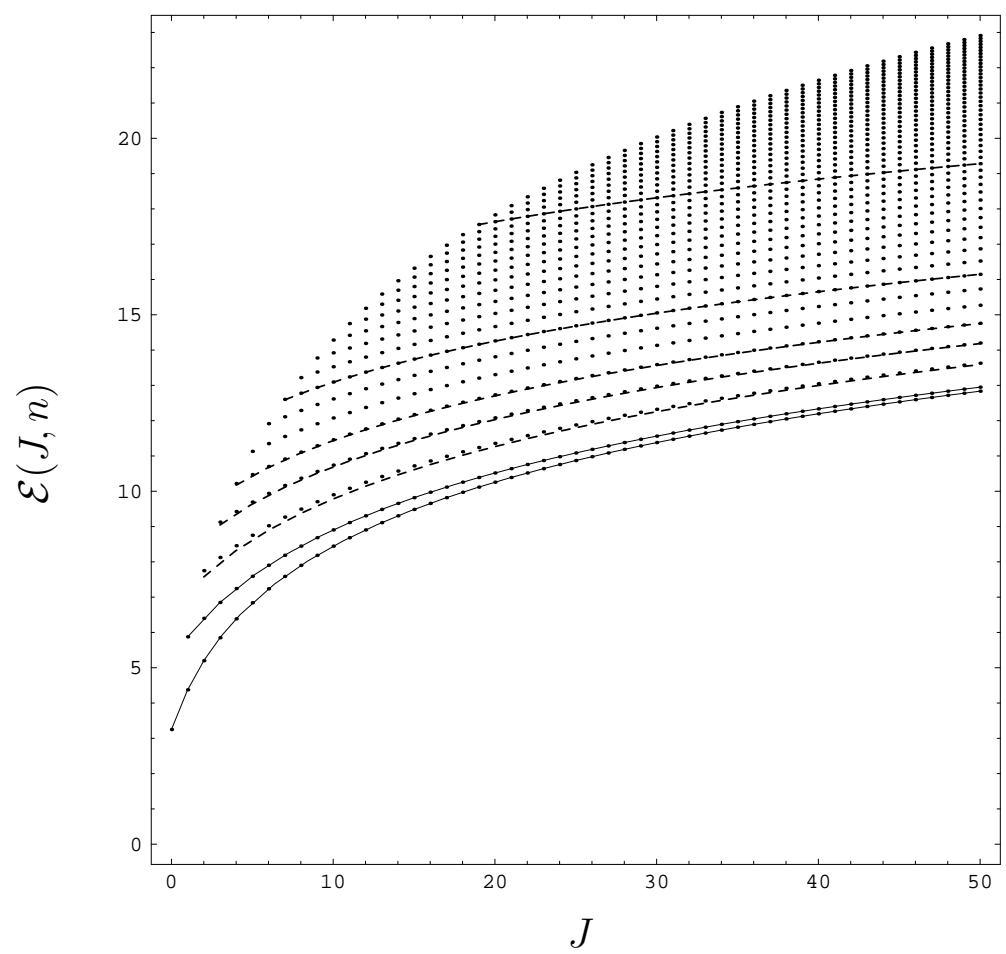

Figure 3: The energy spectrum and the trajectories (in dashed) defined by Eq. (89). The lowest two eigenvalues (solid curves) are given by (88).

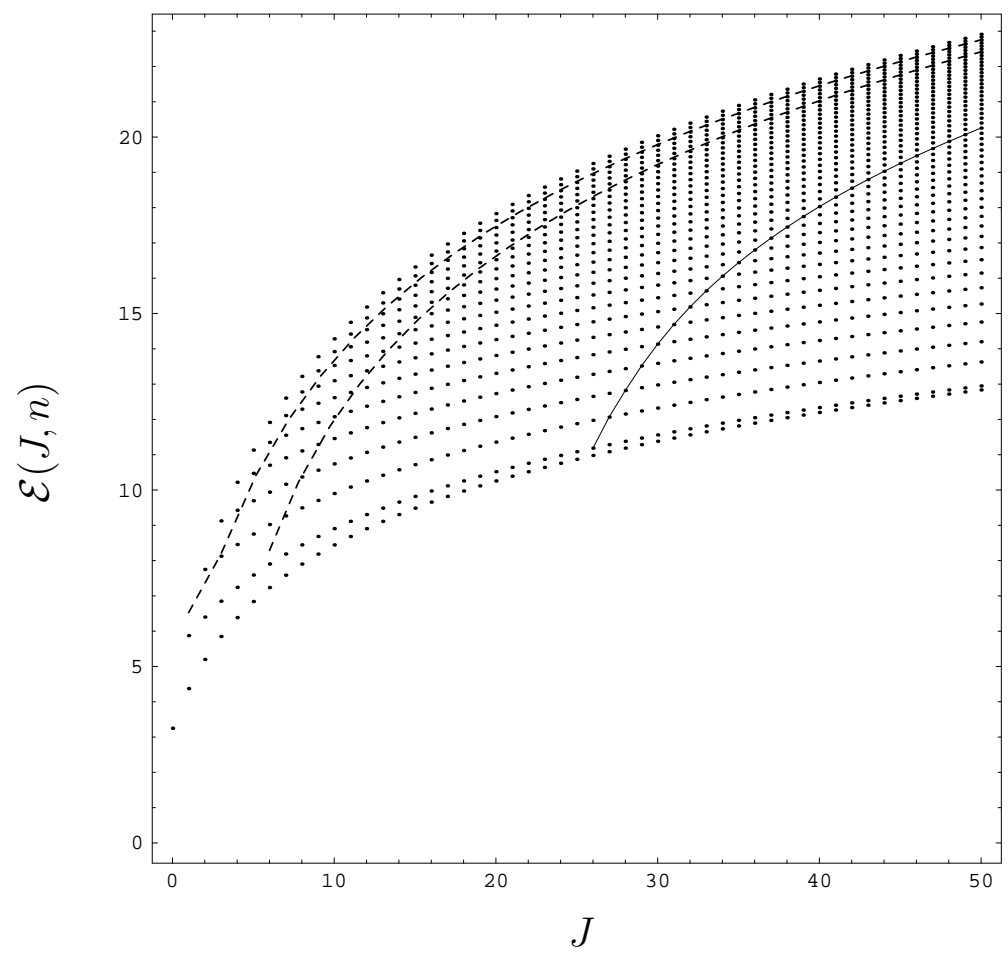

Figure 4: The spectrum of energy and a few (dashed) trajectories described by the analytical formulae of section 7.2 . 
handling [35, 11]

$$
\frac{2}{N_{c}} \mathcal{H}_{j k}=\delta_{j k}\left(\epsilon(j)-\frac{3}{4}\right)-\theta_{j, k+1} \frac{(k+2)(k+3)}{(j-k)(j+2)(j+3)}+\left(\begin{array}{l}
j \rightarrow J-j \\
k \rightarrow J-k
\end{array}\right) .
$$

Here we have used the following step functions $\theta_{j, k}=\{1$, if $j \geq k ; 0$, if $j<k\}$.

The result of numerical diagonalization is presented in Figs. 3 and 4 together with trajectories derived from the study of the master equation. As we have mentioned above the Eq. (89) gives a perfect description for the entire spectrum of the anomalous dimensions. While for the top of the spectrum, due to limited number of terms taken in the WKB expansion of the energy, we cannot penetrate too far inside the spectrum since otherwise the condition $J \gg n$, - whose validity was assumed in the reduction of the recursion relation to the set of differential equations (80,81), will be violated.

\section{Conclusions.}

Making use of the algebraic operator formalism of the Quantum Inverse Scattering Method we have identified an integrable one-dimensional inhohmogeneous three-site open quantum spin chain model to describe the evolution of the twist-three chiral-odd quark-gluon correlators in Quantum Chromodynamics in multicolour limit. The integrability of the evolution equations results into substitution of the original complicated problem of diagonalization of anomalous dimension matrix to the more simple one for the 'hidden' conserved charge. However, since the problem turns out to be still quite complicated to derive the analytical solution we have employed the WKBtype expansion w.r.t. the total conformal spin of the system. Taking the first few non-leading corrections allows to describe the energy spectrum analytically with good accuracy which can be systematically improved, however, at a price of a rather involved analysis. The 'hidden' charge unravel the structure of the spectrum - it distinguishes the particular components of three-parton correlators with different renormalization group dependence.

Once the $1 / N_{c}^{2}$ corrections are taken into account the integrability of the system will be immediately violated. An interesting question is how the trajectories of multicolour anomalous dimensions will mix with each other once the $1 / N_{c}^{2}$ effects are switched on.

The same techniques, up to minor modifications required due to the loss of definite permutation symmetry of quark fields, can be applied to the chiral-even sector corresponding the transverse spin structure function $g_{2}(x)$.

The author was supported by the Alexander von Humboldt Foundation. 


\section{References}

[1] For a review see, B. Lampe, E. Reya, Spin physics and polarized structure functions, hep$\mathrm{ph} / 9810270$.

[2] R.L. Jaffe, X. Ji, Phys. Rev. Lett. 67 (1991) 552; Nucl. Phys. B 375 (1992) 527.

[3] Y. Kanazawa, Y. Koike, N. Nishiyama, Phys. Lett. B 430 (1998) 195.

[4] J. Kodaira, K. Tanaka, Prog. Theor. Phys. 101 (1999) 191.

[5] A.V. Belitsky, in Proc. of the XXXI PNPI Winter School on Nuclear and Particle Physics, (St. Petersburg, 1997) p. 369, hep-ph/9703432.

[6] M. Glück, E. Reya, A. Vogt, Z. Phys. C 48 (1990) 471; Z. Phys. C 53 (1992) 127; Z. Phys. C 67 (1995) 433.

[7] A. Ali, V.M. Braun, G. Hiller, Phys. Lett. B 266 (1991) 117.

[8] I.I. Balitsky, V.M. Braun, Y. Koike, K. Tanaka, Phys. Rev. Lett. 77 (1996) 3078.

[9] A.V. Belitsky, Phys. Lett. B 405 (1997) 312.

[10] J. Kogut, D.E. Soper, Phys. Rev. D 1 (1970) 2901.

[11] A.V. Belitsky, D. Müller, Nucl. Phys. B 503 (1997) 279.

[12] A.P. Bukhvostov, G.V. Frolov, L.N. Lipatov, E.A. Kuraev, Nucl. Phys. B 258 (1985) 601.

[13] A.V. Efremov, A.V. Radyushkin, Phys. Lett. B 94 (1980) 245.

[14] S.J. Brodsky, G.P. Lepage, Phys. Rev. D 22 (1980) 2157.

[15] Yu.M. Makeenko, Sov. J. Nucl. Phys. 33 (1981) 440.

[16] Th. Ohrndorf, Nucl. Phys. B 198 (1982) 26.

[17] D. Müller, Phys. Rev. D 49 (1994) 2525.

[18] A.V. Belitsky, D. Müller, Nucl. Phys. B 537 (1999) 397.

[19] A.V. Belitsky, D. Müller, Nucl. Phys. B 527 (1998) 207.

[20] H. Bateman, A. Erdélyi, Higher transcendental functions, V. 2, (Mc Graw-Hill, New York, 1953). 
[21] G. Mack, A. Salam, Ann. Phys. 53 (1969) 174.

[22] V.M. Braun, S.E. Derkachov, A.N. Manashov, Phys. Rev. Lett. 81 (1998) 2020.

[23] V.M. Braun, S.E. Derkachov, G.P. Korchemsky, A.N. Manashov, Nucl. Phys. B (1999) (in print), hep-ph/9902375.

[24] A.V. Belitsky, Phys. Lett. B 453 (1999) 59.

[25] L.N. Lipatov, JETP Lett. 59 (1994) 596; Nucl. Phys. B 548 (1999) 328.

[26] L.D. Faddeev, G.P. Korchemsky, Phys. Lett. B 342 (1995) 311;

G.P. Korchemsky, Nucl. Phys. B 443 (1995) 255; Nucl. Phys. B 462 (1995) 333.

[27] V.E. Korepin, N.M. Bogoliubov, A.G. Izergin, Quantum inverse scattering method and correlation functions, Cambridge Univ. Press, (Cambridge, 1993).

[28] C. Gomés, M. Ruiz-Altaba, G. Sierra, Quantum groups in two-dimensional physics, Cambridge Univ. Press, (Cambridge, 1996).

[29] E.K. Sklyanin, J. Phys. A 21 (1988) 2375.

[30] D.R. Karakhanyan, R. Kirschner, High-energy scattering in gauge theories and integrable spin chains, hep-th/9902031; Conserved currents of three-reggeon interaction, hep-th/9902147.

[31] P.P. Kulish, E.K. Sklyanin, Quantum spectral transform method. Recent developments, in Lecture Notes in Physics, 151 (Springer-Verlag, 1982), p. 61.

[32] E.K. Sklyanin, Quantum inverse scattering method. Selected topics, in Nankai Lectures in Mathematical Physics Quantum groups and integrable systems, ed. Mo-Lin Ge, (World Scientific, Singapore, 1992), p. 63; hep-th/9211111.

[33] P.P. Kulish, N.Yu. Reshetikhin, E.K. Sklyanin, Lett. Math. Phys. 5 (1981) 393.

[34] L.D. Faddeev, in Les Houches Lectures (1995), How algebraic Bethe ansatz works for integrable models, hep-th/9605187.

[35] Y. Koike, K. Tanaka, Phys. Rev. D 51 (1995) 6125;

Y. Koike, N. Nishiyama, Phys. Rev. D 55 (1997) 3068. 